\title{
Mild Traumatic Brain Injury in Children: Ringing the Alert Bell
}

\author{
Shay Menascu ${ }^{\mathrm{a}, \mathrm{c}}$, Shany M. Tshechmer ${ }^{\mathrm{b}}$
}

\begin{abstract}
Despite the high incidence of mild head injuries in children, only recently has there been increasing interest which began when findings from research done in adult patients showed that the effects of mild closed head injuries could interfere significantly with employment and other areas of life. Mild traumatic brain injury (TBI) is a common occurrence around the world and in the United States, its estimated incidence exceeding 1 million injuries per year, with cognitive, emotional, behavioral, and physical impairments as common sequelae. The etiology of these symptoms in individuals with mild TBI is controversial, with hypotheses of postconcussive symptom formation variously ascribing greater or lesser weight to neural damage, psychiatric factors, somatization, malingering, or some combination of these. TBI in children is an enormous problem., as many children will encounter a brain injury. Adolescents and families need to self-monitor symptoms and limit environments or circumstances that exacerbate any symptoms. When symptoms resolve, a gradual progressive return to play is currently recommended. The recurrence risk for subsequent concussions is elevated, but there is limited documentation of the effectiveness of preventative efforts. This review will limit itself to what may refer to as minor traumatic brain injury, primarily in children, with a focus on the assessment and management of this complexity.
\end{abstract}

Keywords: Mild close head injury; Children; Psychological sequels; Acute and chronic interventions

Manuscript accepted for publication April 19, 2011

aPediatric Neurology Unit, Edmond \& Lili Safra Children's Hospital, Sheba Medical Center \& Tel Aviv University Sackler School of Medicine, Tel-Hashomer, Israel

${ }^{\mathrm{b}}$ Soroka Medical Center \& Department of Behavioral Sciences,

Ben-Gurion University of the Negev, Beer-Sheva, Israe

${ }^{\mathrm{c}}$ Corresponding author: Dr. Shay Menascu. Address: Givton 39, P.O. Box

214, Lehavim, Israel. Email: bguac@yahoo.com

doi:10.4021/jnr16w

\section{Introduction}

Accidents, including severe head injuries, are the leading cause of death in infants and children between the ages of 1 to $14[1,2]$. Research and clinical decisions in traumatic brain injury (TBI) are made more difficult by the lack of reliable measures of brain injury severity. Current protocol in emergency departments is to focus on life threatening and moderate TBI patients who need emergency interventions and monitoring. This approach concentrates the majority of the resources on these patients without taking into account the fact that about two-thirds of TBI patients sustain only a mild injures and are usually discharged to their home after only a brief observation period. As there is uncertainty as to the ideal assessment modality for the injured child, and a lack of uniform recommendations for return to activity and school after MTBI, the American Academy of Pediatrics recognized the importance of mild head injury in children and issued management guidelines for treatment of this common disease. However, it failed to address post injury sequel and their management, an omission likely related to the scarcity of available data [3]. In general, it is difficult to quantify mild head injuries in children and adolescents. As many mild head injuries require no medical management, these individuals most likely are not referred to a medical center for a formal evaluation. The difficulty in diagnosing increases as some mild injuries result in physical symptoms which are not specific such as vomiting, nausea and dizziness which are hard to correlate to a previous head injury that may have gone unrecognized. Children and adolescents are at risk for mild head injuries resulting from accidents in the home and on the playground. Child abuse may also be a cause of mild injury, however, the actual frequency of reported child abuse with correlation to mild closed head injury is not known. All of these situations emphasize the degree to which the diagnosis of mild closed head injury is underestimated.

\section{Definitions, Incidence and Epidemiology}

Accurate statistics regarding the incidence and preva- 
lence of closed-head injuries are relatively difficult to obtain. Terms such as "mild", "minor", "moderate", "minimal", and "trivial" are applied to head injuries without precise or universal definitions. This lack makes comparisons among patient populations difficult and interferes with the development of therapeutic guidelines. The incidence appears to vary as a function of injury severity. One of the most commonly employed definitions of TBI is that proposed by the Mild Trauma Brain Injury Committee of the Head Injury Interdisciplinary, Special Interest Group of the American Congress of Rehabilitation Medicine. In which, a patient with mild traumatic brain injury is a person who has had a traumatically induced physiologic disruption of brain function as manifested by at least one of the following: any period of loss of consciousness; any loss of memory for events immediately before or after the accident; any alteration in mental state at the time of the accident (e.g., feeling dazed, disoriented, or confused); focal neurologic deficit(s) that may or may not be transient, but where the severity of the injury does not exceed the following: loss of consciousness of approximately 30 minutes or less; after 30 minutes, an initial Glasgow Coma Scale (GSC) score of 13 to 15; posttraumatic amnesia not greater than 24 hour. The most common and accepted measure of injury severity is the Glasgow Coma Scale [4] on which scores from 13 - 15 represent mild injuries, while scores from $9-12$ are in keeping with moderate injuries, and scores of 8 or less represent severe injuries. A normal score may be seen with intracranial injury in $28 \%$ of patients. Thus, a normal neurologic examination and a normal GCS do not exclude the possibility of a significant head injury. Mild head injury is defined as a situation in which a patient has had a traumatically induced physiological disruption of brain function, as manifested by at least one of the following: 1) any period of loss of consciousness; 2) any loss of memory for events immediately before or after the accident; 3) any alteration in mental state at the time of the accident; 4) focal neurological deficit(s) which may or may not be transient [5]. Injuries where symptoms and physical findings exceed these criteria are considered to be of more than mild severity.

The United States National Coma Data Bank documents that about $85 \%$ of all head injuries requiring medical treatment are mild in nature, about $8 \%$ are moderate and the remaining $6 \%$ are severe [6]. Many mild head injuries do not come to the attention of health care personnel. The incidence is quite likely to be underestimated rather than overestimated. Additional difficulty in determining an accurate incidence of mild injuries is confounded by the inclusion in these statistics of lacerations and contusions of the face and scalp, with no definite impairment of consciousness. The incidence of closed head injuries varies significantly according to different demographic factors including gender, age and socio-economic status. Boys are at considerably higher risk for closed-head trauma than girls. The ratio of boys to girls rises from approximately $1.5: 1$ for preschool children to approximately $2: 1$ for school-age children and adolescents. These changes appear to reflect the sharp increase in head injuries among males and a gradual decrease among females [7]. The incidence of closed-head trauma also varies with age. Data from the United States demonstrate that the incidence is relatively stable from birth to age 5, with injuries occurring in about 160 per 100,000 children in this age group and after age 5, the overall incidence gradually increases until early adolescence and then shows rapid growth, reaching maximal peak of 290 per 100,000 by the age of 18 years [8]. It has also been shown that incidence rates of mild closed head injuries may also vary as a function of family socioeconomic status [9].

\section{Biomechanics of Brain Pathology}

Closed-head trauma can produce brain injuries including both focal and diffuse lesions as well as disruption in brain function at a cellular level. The pathophysiology of head trauma begins at the time of impact regardless its severity, and then can continue for a prolonged period (weeks to months). Although incompletely understood, TBI likely results in axonal injury (commonly through accelerative/decelerative forces). This axonal injury sets in motion a cascade of neurometabolic changes the result of which is an increase in metabolism (as glucose utilization) along with a local decrease in cerebral blood flow. These metabolic changes can promote additional neuronal injury or delay in recovery. Just as recovery from head injury is variable, so is the attenuation of these metabolic changes. A reliable assessment tool to judge the adequacy of recovery is thus vital injuries resulting from head trauma can be classified into two broad categories, primary and secondary. Primary injuries result directly from the trauma itself and may include skull fractures, contusions, lacerations and mechanical injuries to nerve fibers and blood vessels. Secondary injuries arise indirectly from the trauma related to edema, hypoxia, increased intracranial pressure, and hematomas [10]. The causes of mild TBI are the same as in case of those of severe TBI, but with two major exceptions, as assaults and whiplash are both more common in causes of mild TBI than of severe. The severity of an injury can vary depending on whether the head is in motion or in a stationary position when the impact occurs. It is assumed that the head impact velocity is greater in case of high speed crashes, although this relationship is not always constant. Additionally, neurogenetic factors may influence the extent of neural injury produced by mild TBI. Recent studies suggest that carrier status for the apolipoprotein epsilon-4 (ApoE-4) allele may increase risk for poor outcome following TBI, particularly among persons with more severe TBI or repetitive mild TBI [11]. Conventional EEG may be abnormal in as many as $10 \%$ of persons with mild TBI. Findings on conventional EEG in 
this population most often include mild disorganization of the background rhythms and/or a mild excess of slow wave frequencies. Topographic brain electrical activity mapping and quantitative EEG (QEEG) may demonstrate frontal and frontotemporal abnormalities not evident on conventional EEG. When present, these abnormalities are similar in type and location, although of lesser severity, to those seen following severe TBI [12]. Evoked potential and event-related potential (EP and ERP) studies of persons with mild TBI also demonstrate abnormal brain function. The correlations between specific EP/ERP findings and clinical post-concussive symptoms emerge when the electrophysiologic procedures index dysfunction within the neural systems related to those serving the cognitive and behavioral functions in which the person is experiencing impairment. These abnormalities are associated with the function cortical areas involved in the generation of attention and memory. Electrophysiologic abnormalities of these types in patients with post-traumatic attention and memory impairments offer additional support to the hypothesis that mild TBI does in some cases give rise to neurophysiologically-based persistent cognitive impairments [13].

\section{Cognitive and Psychological Sequelae}

A strikingly high rate of neurocognitive deficits was observed in hospitalized population with TBI. Mild closed head injury can produce various deficits, including a decrease in intellectual functioning, language skills, attention and memory, executive function, academic achievement and even different forms of behavioral adjustment. Strictly applied, the term "mild TBI" refers only to the initial injury severity and should not be interpreted unequivocally as suggesting mild outcome severity. Although both the postconcussive syndrome and postconcussive symptoms are most often discussed in the context of mild TBI, these terms and their clinical referents are not synonymous with mild TBI: mild TBI describes a type of injury whereas postconcussive symptoms or syndrome describes a set of problems resulting from TBI, including mild TBI. Postconcussive symptoms may develop following a TBI of any severity, and are generally grouped into three categories: cognitive, physical, and emotional/behavioral. The term "postconcussive syndrome" denotes the development of a constellation of physical, cognitive, and emotional/behavioral post-concussive symptoms. There is little evidence of coupling of symptom resolution following TBI. Few persons with multiple postconcussive symptoms immediately after TBI experience persistence of the entire set of their symptoms over time, and instead maintain only a few, if any, of them into the late post-injury period. Which of these initial symptoms are maintained is also not reliably predictable based on their early occurrence after TBI. Instead, multiple and varied treatments are generally required for the multiple and varied symptoms of these individuals [14]. Long term difficulties following head injuries are reported in language skills. These difficulties are typically measured by linguistic skills testing. Object description and verbal fluency have been shown to be impaired in a high percentage of children after mild closed head injury [14, 15]. These difficulties seem to be sustained even after word acknowledgement and verbal memory return to normal. As these skills are highly important for school performance, it leads to extensive difficulties experienced on return to school [15]. The degree of memory problems after mild TBI correlates well with the severity of the injury. Memory difficulties have been reported on a wide variety of verbal tests, including tests for recognition of memory for words and words listing learning [16]. The attention problems noted in children with closed head injury include poorer response modulation, especially low ability to concentrate as well as a relatively slower reaction time to different stimuli. When compared to a matched group of children without preceding head injuries, these difficulties are more evident in young children with head trauma than the somewhat older group of children with the same type of injury [17]. Post traumatic behavioral changes are frequently reported after a pediatric head injury and include irritability, poor anger control or different forms of attention deficit disorders [18].

\section{Intervention and Outcome}

Intervention in mild head injury should begin with awareness of the possible symptoms. The complexity and multiplicity of postconcussive symptoms, the subtlety of the neurobiological consequences of TBI, and the inescapability of psychosocial influences on outcome following TBI necessitate an approach to the treatment of persons with mild brain injury that begins with a thorough neuropsychiatric evaluation. Caregivers need to be alert to different presenting symptoms of mild TBI which can be easily misdiagnosed and ignored. The clinical presentation is expected to include at least some elements of the classic constellation of postconcussive symptoms and gradual, although sometimes incomplete, symptomatic improvement over time. In the immediate post-injury period, $80 \%-100 \%$ of persons with mild brain injury will describe one or more symptoms reasonably attributable to their injury, most commonly including headache, slowed thinking, and/or impaired attention and memory [19]. Intervention also involves informing and explaining the injury to the family members. In the first 24 hours after the head injury, the outcome is entirely dependent on the development of intracranial complications that may have not been demonstrated during the first assessment $[19,20]$. Intracranial hematomas were reported to occur in about $1 \%$ of patients and $10 \%$ of those individuals will die [20]. Children and adults were uniformly having an excel- 
lent outcome if intracranial hematomas were drained before clinical deterioration occurred. In the absence of acute complications, most of the children with mild head injury make a recovery in a matter of days to weeks [21]. Neurobehavioral symptoms and other neuropsychological deficiencies rarely may persist for months and even years. Previous published studies indicate that the measures of both diffuse and focal lesions can be used to predict outcome. Long term family assistance can come from different resources and can include local support groups or a head injury foundation. Transitioning the child back to school is important. Different studies have shown that a regular classroom program, similar to the one that the injured child was enrolled in prior to the accident, usually can be adapted to meet the new special needs of the child [22]. The range of neuropsychological stresses, weaknesses and different arrays of cognitive deficiencies reported in children after mild brain injury suggest that, even though some may be considered unaffected by their trauma, there may be difficulties in different neuropsychological and behavioral areas to be considered in the child's return to school. Ewing-Cobbs et al. [23] showed that the variability in neuropsychological problems seen in children involved in a traumatic brain injury indicates the need for assessment of the children, no matter what their level of TBI severity. De Kruijk [24] studied the efficiency of bed rest after a mild traumatic brain injury. One hundred seven patients were enrolled. Fifty-four were advised not to take any bed rest and the other 53 patients were advised to take full bed rest for 6 days after the injury. The primary outcome measures were the severity of post-traumatic complications, using a visual analogue scale and a 36 item short form health survey completed at 2 weeks, 3 weeks and 6 months after the trauma. The conclusion was that as a measure to speed up recovery in patients with post-traumatic complication, bed rest is no more effective than no bed rest at all. A study done by Adams [25] looked at the efficiency of mandatory hospital admission after isolated mild closed injury in children who were diagnosed as concussion alone or a concussion with a brief loss of consciousness who were routinely admitted for observation despite a normal neurological exam, negative finding in their head CT and Glasgow Coma Scale of 15 at the time of presentation. They collected their data from the National Pediatric Trauma Registry. One thousand thirty three patients were identified as having closed isolated head injury and Glasgow Coma Scale of 15 at the time of admission. The authors concluded that in this selected children population, the mandatory admission may not be necessary for isolated mild closed head injury with a negative CT and a normal neurological exam [26].

\section{Summary}

Predicting clinical outcomes in children after TBI is highly variable and needs to combine different complex aspects of each case such as the child's pre-injury global psychological state and support after the injury, behavior, family support and even their economical status. The presence of comorbid psychiatric problems such as a major depressive episode, anxiety disorders (including post-traumatic stress disorder), or substance abuse, whether or not these are regarded as etiologically related to the mild TBI, should be treated aggressively using appropriate psychotherapeutic and pharmacologic interventions. Education early after a mild TBI includes the symptoms it produces, the usual time course for resolution of these symptoms, and the potential for long-term difficulties, which may decrease the likelihood of developing persistent postconcussive symptoms. Clinicians should offer validation of the person's experience of symptoms, regardless of their cause, without fostering illness behaviors. This validation is best coupled with the development of individualized and realistic goals for return to major activities. Unfortunately, the majority of treating clinicians have few tools available to help determine when it is appropriate for the individual to return to activities. This, added to the fact that many mild head injuries never come to medical attention, should lead health care professionals to conclude that the incidence of mild head injury is quite likely to be underestimated rather than overestimated, and that is critically important to accurately identify those children that may develop complex neurological symptoms after mild head trauma and by this focus the needed interventions on them. There are relatively few randomized controlled trials of treatments in the TBI population, there is evidence suggesting that when properly applied they may be of benefit for the treatment of memory, attention, executive function, and communication deficits among reasonably high-functioning and well motivated persons with TBI. When pharmacologic therapies are used, the indications and need for ongoing prescriptions should be reviewed, and efforts should be made to eliminate those not affording clear benefits or that are potentially worsening postconcussive symptoms. More studies are required to evaluate the overall quality of life, health care utilization, influence of the family and the effect of school and school performance after a child has a closed head injury. Research utilizing advances in neuroimaging is necessary to understand the underlying neuropathology of mild closed injury and to attempt to correlate these studies with neuropsychological outcome. Prospective longitudinal studies following children post mild TBI over a period of years will be most helpful, allowing movement beyond group characterization. The aim will then be to provide better treatment and adequate support to children recovering from a mild closed head injury.

\section{Disclosure}

All authors certify that he or she has participated sufficiently 
in the intellectual content and authors declare no conflict of interests related to this manuscript.

\section{References}

1. Satz P, Zaucha K, McCleary C, Light R, Asarnow R, Becker D. Mild head injury in children and adolescents: a review of studies (1970-1995). Psychol Bull 1997;122(2):107-131.

2. Bullock R. Head Injury: pathophysiology and management of severe closed injury. Chapman \& Hall Medical $1997 ; 478$.

3. Gillis D. Concussion in football: when is it safe to return to the game. Neurology Today 2004;4(2):62-64.

4. Zhang L, Yang KH, King AI. A proposed injury threshold for mild traumatic brain injury. J Biomech Eng 2004;126(2):226-236.

5. Bijur PE, Haslum M, Golding J. Cognitive outcomes of multiple mild head injuries in children. J Dev Behav Pediatr 1996;17(3):143-148.

6. Asarnow RF, Satz P, Light R, et al. The UCLA Study of Mild Closed Head Injury in Children and Adolescents. Nils RV, Richard JR. Medical, Broman \& ME Michel Co, 1999.

7. Rees PM. Contemporary issues in mild traumatic brain injury. Arch Phys Med Rehabil 2003;84(12):1885-1894.

8. Baker RJ, Patel DR. Sports related mild traumatic brain injury in adolescents. Indian J Pediatr 2000;67(5):317321.

9. Barry CT, Taylor HG, Klein S, Yeates KO. Validity of neurobehavioral symptoms reported in children with traumatic brain injury. Child Neuropsych 1996;2(3):213226.

10. Biegon A, Fry PA, Paden CM, Alexandrovich A, Tsenter J, Shohami E. Dynamic changes in N-methyl-D-aspartate receptors after closed head injury in mice: Implications for treatment of neurological and cognitive deficits. Proc Natl Acad Sci U S A 2004;101(14):5117-5122.

11. McLean AJ, Anderson RWG. Biochemistry of closed head injury. Head Injury 1997;(2):25-27.

12. King NS. Emotional, neuropsychological, and organic factors: their use in the prediction of persisting postconcussion symptoms after moderate and mild head injuries. J Neurol Neurosurg Psychiatry 1996;61(1):75-81.

13. Webbe FM, Barth JT. Short-term and long-term outcome of athletic closed head injuries. Clin Sports Med 2003;22(3):577-592.

14. Putukian M, Echemendia RJ. Psychological aspects of serious head injury in the competitive athlete. Clin Sports Med 2003;22(3):617-630, xi.

15. Ryan LM, Warden DL. Post concussion syndrome. Int Rev Psychiatry 2003;15(4):310-316.

16. Pelinka LE. Serum markers of severe traumatic brain injury. Indian Journal of Critical Care Medicine 2004;8:190-193.

17. Savola O, Pyhtinen J, Leino TK, Siitonen S, Niemela O, Hillbom M. Effects of head and extracranial injuries on serum protein $\mathrm{S} 100 \mathrm{~B}$ levels in trauma patients. J Trauma 2004;56(6):1229-1234; discussion 1234.

18. Levin HS, Hanten G, Zhang L, Swank PR, Ewing-Cobbs L, Dennis M, Barnes MA, et al. Changes in working memory after traumatic brain injury in children. Neuropsychology 2004;18(2):240-247.

19. Yeates KO, Taylor HG. Neurobehavioural outcomes of mild head injury in children and adolescents. Pediatr Rehabil 2005;8(1):5-16.

20. Max JE, Roberts MA, Koele SL, Lindgren SD, Robin DA, Arndt S, Smith WL, Jr., et al. Cognitive outcome in children and adolescents following severe traumatic brain injury: influence of psychosocial, psychiatric, and injury-related variables. J Int Neuropsychol Soc 1999;5(1):58-68.

21. Fletcher JM, Levin HS, Lachar D, Kusnerik L, Harward H, Mendelsohn D, Lilly MA. Behavioral outcomes after pediatric closed head injury: relationships with age, severity, and lesion size. J Child Neurol 1996;11(4):283290.

22. Korinthenberg R, Schreck J, Weser J, Lehmkuhl G. Post-traumatic syndrome after minor head injury cannot be predicted by neurological investigations. Brain Dev 2004;26(2):113-117.

23. Ewing-Cobbs L, Miner ME, Fletcher JM, Levin HS. Intellectual, motor, and language sequelae following closed head injury in infants and preschoolers. J Pediatr Psychol 1989;14(4):531-547.

24. De Kruijk JR, Leffers P, Menheere PP, Meerhoff S, Rutten J, Twijnstra A. Prediction of post-traumatic complaints after mild traumatic brain injury: early symptoms and biochemical markers. J Neurol Neurosurg Psychiatry 2002;73(6):727-732.

25. Adams J, Frumiento C, Shatney-Leach L, Vane DW. Mandatory admission after isolated mild closed head injury in children: is it necessary? J Pediatr Surg 2001;36(1):119-121.

26. Simon B, Letourneau P, Vitorino E, McCall J. Pediatric minor head trauma: indications for computed tomographic scanning revisited. J Trauma 2001;51(2):231237; discussion 237-238. 